\title{
Association between Perfluoroalkyl substances and thyroid stimulating hormone among pregnant women: a cross-sectional study
}

\author{
Yan Wang ${ }^{1 *}$, Anne P Starling ${ }^{1,2}$, Line S Haug ${ }^{3}$, Merete Eggesbo ${ }^{4}$, Georg Becher ${ }^{3,5}$, Cathrine Thomsen ${ }^{3}$, \\ Gregory Travlos ${ }^{1}$, Debra King ${ }^{1}$, Jane A Hoppin ${ }^{1}$, Walter J Rogan ${ }^{1}$ and Matthew P Longnecker ${ }^{1}$
}

\begin{abstract}
Background: Perfluoroalkyl substances (PFASs) are a group of highly persistent chemicals that are widespread contaminants in wild life and humans. Exposure to PFAS affects thyroid homeostasis in experimental animals and possibly in humans. The objective of this study was to examine the association between plasma concentrations of PFASs and thyroid stimulating hormone (TSH) among pregnant women.

Methods: A total of 903 pregnant women who enrolled in the Norwegian Mother and Child Cohort Study from 2003 to 2004 were studied. Concentrations of thirteen PFASs and TSH were measured in plasma samples collected around the $18^{\text {th }}$ week of gestation. Linear regression models were used to evaluate associations between PFASs and TSH.

Results: Among the thirteen PFASs, seven were detected in more than $60 \%$ of samples and perfluorooctane sulfonate (PFOS) had the highest concentrations (median, $12.8 \mathrm{ng} / \mathrm{mL}$; inter-quartile range [IQR], $10.1-16.5 \mathrm{ng} / \mathrm{mL}$ ). The median TSH concentration was $3.5(\mathrm{IQR}, 2.4-4.8) \mu \mathrm{IU} / \mathrm{mL}$. Pregnant women with higher PFOS had higher TSH levels. After adjustment, with each $1 \mathrm{ng} / \mathrm{mL}$ increase in PFOS concentration, there was a $0.8 \%$ (95\% confidence interval: $0.1 \%, 1.6 \%)$ rise in TSH. The odds ratio of having an abnormally high TSH, however, was not increased, and other PFASs were unrelated to TSH.
\end{abstract}

Conclusions: Our results suggest an association between PFOS and TSH in pregnant women that is small and may be of no clinical significance.

Keywords: Perfluorinated alkyl substances, Thyroid stimulating hormone, Pregnant women, The Norwegian Mother and child cohort study, MoBa

\section{Background}

Perfluoroalkyl substances (PFASs) are a group of man-made chemicals with a fully fluorinated hydrophobic linear carbon chain attached to a hydrophilic functional group [1]. Being both lipo- and hydrophobic, they are widely used in industry to make products that are resistant to heat, oil, stains, grease and water [2]. Furthermore, the very high strength of the carbon-fluoride bonds makes PFASs highly stable and resistant to environmental degradation [3]. As

\footnotetext{
* Correspondence: wangy13@niehs.nih.gov

'Department of Health and Human Services, National Institute of Environmental Health Sciences, National Institutes of Health, Durham, NC, USA

Full list of author information is available at the end of the article
}

a consequence, they are widespread in the environment, wildlife, and humans [4]. Diet is thought to be the main exposure route of PFASs for humans [5,6], and ingestion of dust from indoor environments may also contribute to the internal dose [7]. PFASs may act as endocrine disruptors by altering the activity of endogenous hormones [8].

Thyroid hormones play an essential role in ensuring a healthy pregnancy and normal fetal growth and development. Fetuses depend on the mother's supply of T4 entirely during the first trimester, and continue to rely on it to varying degrees throughout the pregnancy $[9,10]$. At birth, around $30 \%$ of thyroid hormone is from the mother [11]. Alterations of maternal thyroid hormone

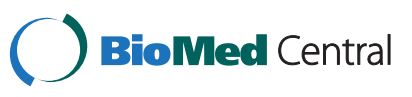

(c) 2013 Wang et al.; licensee BioMed Central Ltd. This is an Open Access article distributed under the terms of the Creative Commons Attribution License (http://creativecommons.org/licenses/by/2.0), which permits unrestricted use, distribution, and reproduction in any medium, provided the original work is properly cited. 
status, even mild, are associated with adverse outcomes for the mother and offspring [12,13].

In pregnant rats and their offspring, treatment with perfluorooctane sulfonate (PFOS) can cause a decrease in total plasma thyroxine (T4) and triiodothyronin (T3) [14-17]. In humans, some PFASs have been associated with elevated T4 levels [18-20] and higher exposure to perfluorooctanoic acid (PFOA) or PFOS was related to increased risk of thyroid disease [21]. Other studies, however, did not find any association between PFASs and thyroid function [22-24]. Findings have generally been inconsistent and most studies were carried out in non-pregnant adults. One study on PFAS in pregnancy, however, showed an association of maternal pregnancy serum PFOA with increased cord TSH [25].

Given the potential association between PFASs and thyroid hormones, concern arises about whether PFAS exposure may disrupt maternal thyroid hormone homeostasis during pregnancy. In the present study, our aim was to examine the relationship between PFASs and TSH as a screening measure of thyroid dysfunction in pregnant women.

\section{Methods}

\section{Study design}

The Norwegian Mother and Child Cohort Study (MoBa) is a prospective population-based pregnancy cohort study conducted by the Norwegian Institute of Public Health [26]. Participants were recruited from all over Norway from 1999-2008, and 38.5\% of invited women consented to participate. They received a postal invitation and the baseline questionnaire in weeks 13-17 of pregnancy, together with an appointment for an ultrasound scan in week 17-18 of pregnancy. The Regional Committee for Medical Research and the Norwegian Data Inspectorate approved the study, and signed informed consent was obtained from each participant.

The subjects in the present study were from a previous case-control analysis of subfecundity in a subset of $\mathrm{MoBa}$ women [27]. These subjects $(\mathrm{N}=950)$ were selected from MoBa participants who enrolled in 2003 and 2004, delivered a live born child and provided a blood sample around week 17-18 of gestation. Among these eligible women, 400 subfecund women (who planned their pregnancy and reported time to pregnancy $>12$ months) were selected randomly. Additionally, 550 control women were selected at random. More details can be found in the previous publication [27]. Twenty of the 950 women reported a thyroid abnormality and were excluded from the main analyses.

\section{Assessment of PFASs}

Blood specimens were collected at enrollment [28], and the mean gestational age at blood draw was 18 weeks.
Up to thirteen PFASs were measured in plasma using high-performance liquid chromatography/tandem mass spectrometry at the Norwegian Institute of Public Health; these were PFOS, PFOA, perfluoroheptanoic acid (PFHpA), perfluorononanoic acid (PFNA), perfluorodecanoic acid (PFDA), perfluoroundecanoic acid (PFUnDA), perfluorododecanoic acid (PFDoDA), perfluorotridecanoic acid (PFTrDA), perfluorotetradecanoic acid (PFTeDA), perfluorobutanoic acid (PFBA), perfluorohexane sulfonic acid (PFHxS), perfluoroheptane sulfonic acid (PFHpS), and perfluorooctane sulfonamide (PFOSA). A detailed description of the analytic method has been published [29]. Among the substances measured, PFOA, PFOS, PFDA, PFHpS, PFHxS, PFNA, and PFUnDA were detected in more than $69 \%$ of samples; the six other PFASs were detected in less than $25 \%$ of the samples and were not included in the subsequent analysis. The limit of quantification (LOQ) was $0.1 \mathrm{ng} / \mathrm{ml}$ for PFBA and $0.05 \mathrm{ng} / \mathrm{ml}$ for all others. For the seven most frequently detected PFASs, values below the LOQ were replaced by the LOQ divided by the square root of 2 . The intra-assay coefficients of variation (CVs) of all seven PFASs were $<10 \%$ except PFUnDA (13.1\%), and the inter-assay CVs of all seven PFASs were $<15 \%$ except PFUnDA (18.7\%) and PFHpS (25.2\%).

\section{Assessment of TSH}

Plasma TSH measurements were performed on the same plasma specimen using an immunoassay (MILLIPLEX map kit, Millipore Corporation, Billerica, MA, USA) on the LiquiChip 200 Workstation (Qiagen Inc., Germantown, MD) at the National Institute of Environmental Health Sciences, USA. Duplicate plasma TSH concentrations were measured for each sample and the mean was used in the analysis. The minimum detectable concentration was $0.01 \mu \mathrm{IU} / \mathrm{mL}$. Both the intra-assay CV and inter-assay CV were $<10 \%$.

\section{Statistical analysis}

The main outcome, TSH, was natural logarithm transformed (ln-transformed) to obtain a normal distribution. As there was no statistically significant difference in mean ln-TSH concentrations between the subfecund subjects and the control subjects (1.22 vs $1.19 \mu \mathrm{IU} / \mathrm{mL}$, student t-test $P=0.34$ ), we combined them in the subsequent analysis. Scatter plots showed approximately linear relationships between PFASs and ln-TSH, thus we used linear regression models to examine PFASs and $\ln -\mathrm{TSH}$ and applied weighted methods (PROC SURVEYMEANS, PROC SURVEYREG and SURVEYLOGISTIC in SAS 9.2, Cary, NC, USA) to adjust for selection into the previous case-control study [30]. Each PFAS was examined in a separate model and was represented either as a continuous variable, or categorically based on quartiles. Logistic 
regression models were also used to examine TSH dichotomized as at or above the $95^{\text {th }}$ percentile $(7.5 \mu \mathrm{IU} / \mathrm{mL})$ or below.

We identified potentially confounding factors based on the literature $[6,25,31,32]$. They included age, gestational age at blood draw, pre-pregnancy body mass index $\left(\mathrm{BMI}, \mathrm{kg} / \mathrm{m}^{2}\right)$, parity, smoking during pregnancy, interval between previous birth and current pregnancy, duration of breast-feeding a previous child, total seafood intake at mid-pregnancy, and plasma concentrations of high-density lipoprotein (HDL) and albumin. With the exception of age and gestational age, which were included a priori, confounders for inclusion in the models were identified using bivariate linear regression models with the exposure and outcome variables. Only those variables associated with any PFAS and also with TSH $(P<0.1)$ were included in the final models. Twenty-seven women were excluded because of missing data on covariates. Additional adjustment for consumption of fatty fish or use of medications that can affect thyroid hormone levels had essentially no effect on the results (not shown). In a sensitivity analysis we also fit a logistic model of self-reported thyroid abnormality during pregnancy $(n=20)$ or not $(n=903)$ in relation to each PFAS. We also repeated the linear regression models of TSH after stratifying according to whether the women were in the control or subfecund group.

\section{Results}

\section{Study population and plasma concentrations of PFASs and TSH}

The average maternal age was 30 years (standard deviation $[\mathrm{SD}]=4$ ) and ranged from 18 to 44 years (Table 1). Almost all of the women (97\%) were in the second trimester at the time of the blood draw (average, 18 weeks; range, 12-37 weeks). Half of the women were nulliparous.

Among the seven most frequently detected PFASs, the median concentration was greatest for PFOS $(12.8 \mathrm{ng} / \mathrm{mL}$; inter-quartile range [IQR], $10.1-16.5 \mathrm{ng} / \mathrm{mL}$ ), followed by PFOA, PFHxS, PFNA, PFUnDA, PFHpS, and PFDA (Table 2). Moderate positive correlations were found between concentrations of PFOS and PFOA $(r=0.66$, $P<0.001)$, and between PFOS and PFHpS $(r=0.69$, $P<0.001)$. The median blood concentration of TSH was 3.5 (IQR: 2.4, 4.8) $\mu \mathrm{IU} / \mathrm{mL}$.

Based on bivariate linear regression models, all PFAS concentrations in nulliparous women were higher than in parous women $(P<0.1)$. Among parous women, those who experienced longer inter-pregnancy interval had higher concentrations of PFASs, except PFHxS $(P<0.05)$. Most PFASs (all except PFHxS and PFOA) were positively associated with seafood intake in times/ week $(P<0.05)$.
Table 1 Characteristics of the 903 subjects

\begin{tabular}{lcc}
\hline & Mean (SD) & Range \\
\hline Age (yrs) & $30(4)$ & $18-44$ \\
Gestational age (weeks) & $18(2)$ & $12-37$ \\
Pre-pregnancy BMI $\left(\mathrm{kg} / \mathrm{m}^{2}\right)$ & $25(7)$ & $15-45$ \\
$\mathrm{HDL}(\mathrm{mg} / \mathrm{dL})$ & $67(12)$ & $39-106$ \\
Total seafood intake (times/week) & $5(5.9)$ & $0-56$ \\
Inter-pregnancy interval (months) & $48(37)$ & $0-245$ \\
Duration of breast-feeding a & $8(6)$ & $0-36$ \\
previous child (months) & $\%$ & \\
& & \\
Parity & & \\
$\quad$ Nulliparous & 50 & \\
Parous & 50 & \\
Smoking during pregnancy & & \\
$\quad$ No & 76 & \\
$\quad$ Yes & 24 & \\
Maternal education & & \\
$<$ High school & & \\
High school & & \\
Some college & & \\
\hline
\end{tabular}

\section{Associations between PFASs and TSH}

Among the seven PFASs, PFHpS, PFNA, and PFOS were positively associated with TSH levels (Table 3). Adjustment for confounders reduced the magnitude and statistical significance of associations between PFHpS and TSH, and PFNA and TSH. Only PFOS remained statistically significant after adjustment $(P=0.03)$, with a slight attenuation in the coefficient $(\beta)$. To express the coefficients as percent change in TSH (original units), per $\mathrm{ng} / \mathrm{mL}$ increase in PFOS, we calculated $100 \times[\exp (\beta)-1]$. For example, the adjusted $\beta$ of 0.008 for PFOS means a $100 \times[\exp$ $(0.008)-1]$ or $0.8 \%(95 \% \mathrm{CI}:[0.1 \%, 2.0 \%])$ increase in $\mathrm{TSH}$ per $\mathrm{ng} / \mathrm{mL}$ increase in PFOS.

Next, to examine the relationship between categorical PFASs and TSH levels we divided PFASs into quartiles and used the first quartile as the reference group. Figure 1 shows that there was an increasing trend in TSH across the quartiles of PFOS, but only women in the third and fourth quartiles had significantly higher TSH levels compared with the reference group.

When TSH was dichotomized (TSH $\geq 7.5 v s<7.5 \mu \mathrm{IU} /$ $\mathrm{mL}$ ) in logistic models, no significant association with any PFAS emerged, whether the latter was considered either as a continuous or categorical variable. In addition, there were no significant associations found between PFASs and self-reported thyroid abnormality.

Among the women who were selected as controls from the MoBa cohort, we observed very similar results. The 
Table 2 Distributions of plasma concentrations of PFASs $(\mathrm{ng} / \mathrm{mL})$ and TSH $(\mu \mathrm{IU} / \mathrm{mL})$

\begin{tabular}{|c|c|c|c|c|c|c|}
\hline PFASs * & $\%>$ LOQ & Geometric mean $(95 \% \mathrm{Cl})$ & $25^{\text {th }}$ percentile & Median & $75^{\text {th }}$ percentile & Maximum \\
\hline PFDA & 69 & $0.09(0.08,0.09)$ & 0.04 & 0.09 & 0.15 & 1.77 \\
\hline PFHpS & 88 & $0.12(0.11,0.12)$ & 0.08 & 0.13 & 0.18 & 0.97 \\
\hline PFHXS & 99 & $0.62(0.59,0.64)$ & 0.43 & 0.60 & 0.84 & 21.74 \\
\hline PFNA & 99 & $0.37(0.36,0.39)$ & 0.28 & 0.39 & 0.51 & 3.01 \\
\hline PFOA & 100 & $2.13(2.07,2.20)$ & 1.57 & 2.15 & 2.95 & 13.99 \\
\hline PFOS & 100 & $12.77(12.45,13.10)$ & 10.13 & 12.81 & 16.49 & 104.18 \\
\hline PFUnDA & 94 & $0.20(0.19,0.21)$ & 0.14 & 0.22 & 0.32 & 1.17 \\
\hline TSH & 100 & $3.39(3.25,3.49)$ & 2.44 & 3.52 & 4.81 & 18.64 \\
\hline
\end{tabular}

${ }^{*} \mathrm{~N}=903$. For values of PFASs below LOQ, LOQs divided by square root of 2 were imputed and used in the calculations of statistics (PROC SURVEYMEANS).

only significant association found was between PFOS and TSH (adjusted $\beta=0.008$ [95\% CI: 0.001, 0.016]). Among women in the subfecund group, the association was inverse and non-significant $(\beta=-0.0016,95 \% \mathrm{CI}$ : $[-0.008,0.005])$.

\section{Discussion}

The precise classification of suboptimal thyroid status requires measurement of more than one hormone. For example, low free T4 with normal TSH reflects hypothyroxinemia [33]. Elevated TSH with normal free T4 reflects subclinical hypothyroidism. In the present study, where we used TSH by itself as a screening measure, we saw no indication that PFOS was associated with abnormally high TSH. Rather, the data suggested an association within the normal range of TSH, and in the absence of a measure of free T4, the clinical importance is unclear. Because we saw no association with abnormally high TSH nor with the presence of a history of thyroid disease, our generally null results were reassuring and consistent with a subtle association that may reflect only a biochemical curiosity. However, the possibility exists that our study did not have enough statistical power to detect a small association with abnormally high TSH, and that there is a small effect on public health (increased hypothyroidism).

Table 3 Unadjusted and adjusted $\beta$ and $95 \%$ confidence intervals for associations between PFASs and TSH (In-transformed)

\begin{tabular}{lcc}
\hline PFASs $(\mathbf{n g} / \mathbf{m L})$ & Unadjusted $\boldsymbol{\beta}(\mathbf{N}=\mathbf{9 0 3})$ & Adjusted $\boldsymbol{\beta}(\mathbf{N}=\mathbf{9 0 3})^{\text {* }}$ \\
\hline PFDA & $0.246(-0.258,0.749)$ & $0.060(-0.458,0.578)$ \\
PFHpS & $0.537(0.142,0.932)$ & $0.299(-0.113,0.710)$ \\
PFHxS & $0.026(-0.031,0.084)$ & $0.013(-0.043,0.070)$ \\
PFNA & $0.251(0.070,0.432)$ & $0.165(-0.023,0.353)$ \\
PFOA & $0.029(-0.008,0.066)$ & $-0.0001(-0.045,0.044)$ \\
PFOS & $0.0120 .005,0.019)$ & $0.008(0.001,0.016)$ \\
PFUnDA & $0.194(-0.069,0.457)$ & $0.080(-0.200,0.360)$ \\
\hline
\end{tabular}

* Models adjusted for maternal age, gestational age at blood draw, HDL concentrations, total seafood intake, parity and inter-pregnancy interval.
In a study of 44 Korean pregnant women with serum measures of PFAS and several thyroid hormones in cord blood [25], inverse associations were found between maternal PFOS and neonatal total T3, and between maternal perfluorotridecanoic acid and neonatal total T3 and T4. In addition, maternal PFOA was associated with increased cord TSH $(\mathrm{n}=31)$. However, the median maternal PFOS concentration (2.93 [IQR: 2.08, 4.36] $\mathrm{ng} / \mathrm{mL}$ ) was much lower than that in our study. In a case-control study of hypothyroxinemia among Canadian pregnant women (96 cases, 175 controls), the authors found no association with serum concentrations of PFOA, PFOS and PFHxS. In that study, the geometric mean of PFOS (7.39 ng/mL) [23] was lower than among women in the present study $(12.77 \mathrm{ng} / \mathrm{mL})$.

In animal experiments, treatment with PFAS generally does not affect serum TSH levels [14,34]. When effects

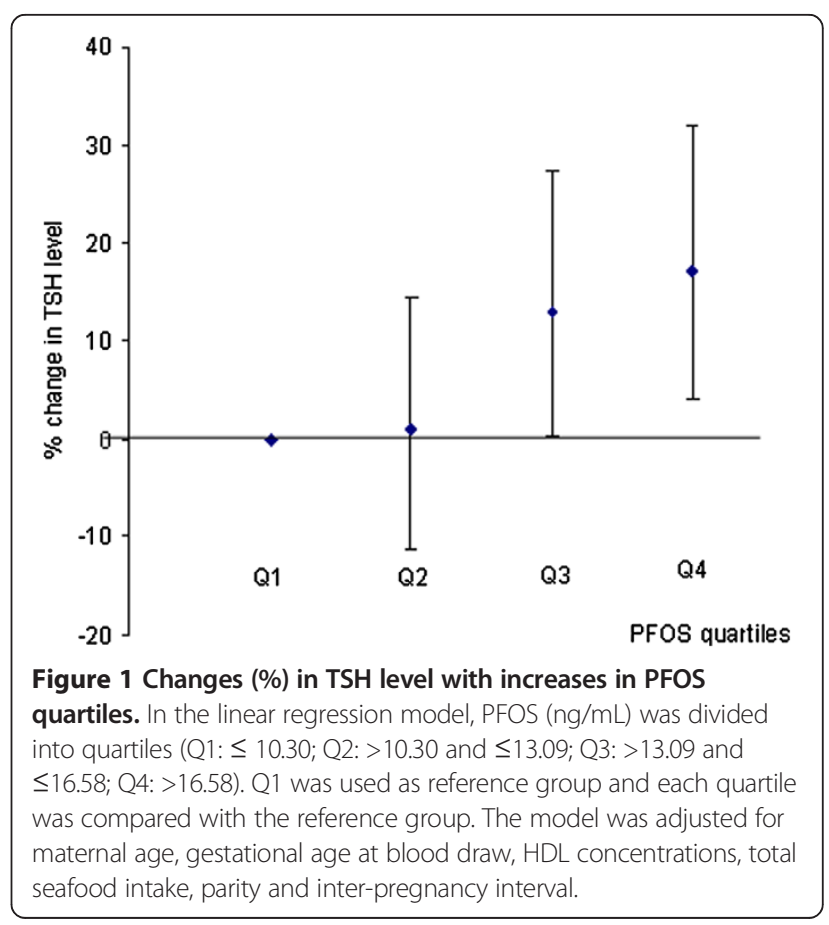


on TSH are seen, they tend to be transient decreases after dosing [17]. PFASs appear to displace thyroxine from transthyretin, causing a transient increase in free T4 [35]. The end effect may be a reduced total T4 with adequate free T4 [14]. Data from animal models also indicate other potential mechanisms by which PFAS may affect thyroid hormone metabolism [34]. Thyroid metabolism in rodents, however, is different than in humans, partly because of transthyretin being a more important transport protein in rodents. Furthermore, during human pregnancy, human chorionic gonadotropin and estrogen affect the TSH feedback mechanism [36], further complicating comparison across species.

Compared with women from other western countries, the median concentrations of PFOS and PFOA in this study were slightly lower $[21,37,38]$. For TSH levels, the median in our study was higher than that in other studies of women in the second trimester $[39,40]$. The difference may explained by the use of different immuno-metric assays. TSH levels can differ by as much as $1.0 \mu \mathrm{IU} / \mathrm{mL}$ because different monoclonal antibodies may recognize different TSH isoforms circulating in the blood $[36,41]$. Iodine deficiency also affects TSH levels, but is rare in Norway [42]. The prevalence of self-reported thyroid disease in the present study (2.2\%) was a little higher than that among pregnant women in another recent study from Norway [43].

We found concentrations of PFOA and PFHxS were both correlated with PFOS, suggesting they might come from common sources. However, they were not associated with TSH levels statistically and PFOA showed a different direction of association. These substances were usually present lower at levels than PFOS, and have different binding capacities with transthyretin due to different structures [35].

The observed association between PFOS and TSH was not present in the subfecund women. Although thyroid disorders are related to reduced fertility [44], we did not find a significant difference in TSH levels between subfecund women and controls. Subfecundity may mask the association, if any, between PFASs and TSH levels.

Our study had several limitations. Unfortunately, we did not have access to sufficient plasma volume to perform any other measurements of thyroid hormones, such as total T4, free T4 and T3. TSH, however, is a sensitive marker of thyroid status and reflects the physiologic log-linear relationship of TSH to free T4 [36], and is often used by itself in screening tests. Plasma TSH levels can reflect mild thyroid functional impairment even when plasma T4 and T3 concentrations are within normal ranges [45]. Second, because our study was cross-sectional study, reverse causation could affect the results. For example, if low thyroid function led to reduced glomerular filtration rate [46], this could increase circulating PFAS concentrations [47]. Third, as TSH levels change during pregnancy, a single measurement may not adequately characterize thyroid homeostasis during pregnancy. Fourth, although we considered some known potential confounders, there may be unmeasured confounders not taken into account. For example, the effects of other chemicals, such as polychlorinated biphenyls (PCBs), on $\mathrm{TSH}$, can not be ruled out, given that fish intake is a source of both PFASs and PCBs. Finally, the participation rate in MoBa was low, raising the possibility of selection bias. Nilsen et al. compared general characteristics, exposures, pregnancy complications, and birth outcomes between MoBa participants and all women giving birth in Norway and found differences in prevalence estimates in most variables (Additional file 1: Table S1) [48]. For the subjects in the present study we tabulated their characteristics using the same format that Nilsen et al. used (Additional file 1: Table S1). The present subjects were generally similar to all MoBa participants, though some minor differences were apparent, as would be expected due to the subgroup sampled because of a long time-to-pregnancy. In spite of the differences between all women giving birth in Norway and MoBa participants, Nilsen et al. found estimates of exposure-outcome associations were similar in the whole population and the MoBa participants [48], and we expect this generalization holds for the present analysis, in which inverse-sampling weights were used.

\section{Conclusions}

Our results suggest an association between PFOS and $\mathrm{TSH}$ in pregnant women that is small and maybe of no clinical significance.

\section{Additional file}

Additional file 1: Table S1. Background characteristics, pregnancyrelated exposures, and pregnancy complications [N(\%)] for all women giving birth in Norway, MoBa participants, and subjects in the present study ${ }^{*}$

\section{Abbreviations}

BMl: Body mass index; Cl: Confidence interval; CV: Coefficient of variation; HDL: High density lipoprotein; IQR: Inter-quartile range; LOQ: Limit of quantification; MoBa: The Norwegian Mother and child cohort study; PCBs: Polychlorinated biphenyls; PFASs: Perfluoroalkyl substances; PFBA: Perfluorobutanoic acid; PFDA: Perfluorodecanoic acid; PFDoDA: Perfluorododecanoic acid; PFHpA: Perfluoroheptanoic acid; PFHpS: Perfluoroheptane sulfonic acid; PFHxS: Perfluorohexane sulfonic acid; PFNA: Perfluorononanoic acid; PFOA: Perfluorooctanoic acid; PFOS: Perfluorooctane sulfonate; PFOSA: Perfluorooctane sulfonamide; PFTeDA: Perfluorotetradecanoic acid; PFTrDA: Perfluorotridecanoic acid; PFUnDA: Perfluoroundecanoic acid; T3: Triiodothyronine; T4: Thyroxine; TSH: Thyroid stimulating hormone.

\section{Competing interests}

The authors declare that they have no competing interests. 


\section{Authors' contributions}

MPL developed the study design and conducted the study; YW performed data analysis and drafted the manuscript; WJR and APS participated in reviewing, editing, and revising the manuscript; $L S H, M E, G B$, and $C T$ contributed to the assessment of PFASs; GT and DK contributed to the assessment of TSH; and JAH assisted in the biobank of MoBa. All authors read and approved the final manuscript.

\section{Acknowledgements}

This study was supported in part by the Intramural Research Program, National Institute of Environmental Health Sciences (NIEHS), National Institutes of Health (NIH). Ms. Starling was supported by an extramural award (1-F30-ES022126-01) from the National Institute of Environmental Health Sciences. The Norwegian Mother and Child Cohort Study was supported by the Norwegian Ministry of Health and the Ministry of Education and Research, NIH/NIEHS (Contract No. N01-ES-85433), NIH/NINDS (Grant No.1 UO1 NS 047537-01), and the Norwegian Research Council/FUGE (Grant No. 151918/S10). We are grateful to all the participating families in Norway who take part in this ongoing cohort study.

\section{Author details}

${ }^{1}$ Department of Health and Human Services, National Institute of Environmental Health Sciences, National Institutes of Health, Durham, NC, USA. ${ }^{2}$ Department of Epidemiology, The University of North Carolina at Chapel Hill, Chapel Hill, NC, USA. ${ }^{3}$ Department of Environmental Medicine, Norwegian Institute of Public Health, Oslo, Norway. ${ }^{4}$ Department of Genes and Environment, Division of Epidemiology, Norwegian Institute of Public Health, Oslo, Norway. ${ }^{5}$ Department of Chemistry, University of Oslo, Oslo, Norway.

Received: 17 April 2013 Accepted: 3 September 2013

Published: 8 September 2013

\section{References}

1. Kantiani L, Llorca M, Sanchís SJ, Farre M, Barcelo D: Emerging food contaminants: a review. Environ Sci Technol 2010, 398:2413-2427.

2. Renner R: Growing concern over perfluorinated chemicals. Environ Sci Technol 2001, 35:154A-160A

3. Johnson JD, Gibson SJ, Ober RE: Cholestyramine-enhanced fecal elimination of carbon-14 in rats after administration of ammonium [14C] perfluorooctanoate or potassium [14C]perfluorooctanesulfonate. Fundam Appl Toxicol 1984, 4:972-976.

4. Giesy JP, Kannan K: Global distribution of perfluorooctane sulfonate in wildlife. Environ Sci Technol 2001, 35:1339-1342.

5. Fromme H, Schlummer M, Moller A, Gruber L, Wolz G, Ungewiss J, Bohmer S, Dekant W, Mayer R, Liebl B, Twardella D: Exposure of an adult population to perfluorinated substances using duplicate diet portions and biomonitoring data. Environ Sci Technol 2007, 41:7928-7933.

6. Haug LS, Thomsen C, Brantsaeter AL, Kvalem HE, Haugen M, Becher G, Alexander J, Meltzer HM, Knutsen HK: Diet and particularly seafood are major sources of perfluorinated compounds in humans. Environ Int 2010, 36:772-778

7. Haug LS, Huber S, Becher G, Thomsen C: Characterisation of human exposure pathways to perfluorinated compounds-comparing exposure estimates with biomarkers of exposure. Environ Int 2011, 37:687-693.

8. White SS, Fenton SE, Hines EP: Endocrine disrupting properties of perfluorooctanoic acid. J Steroid Biochem Mol Biol 2011, 127:16-26.

9. Contempre B, Jauniaux E, Calvo R, Jurkovic D, Campbell S, de Escobar GM: Detection of thyroid hormones in human embryonic cavities during the first trimester of pregnancy. J Clin Endocrinol Metab 1993, 77:1719-1722.

10. Vulsma T, Gons MH, de Vijlder JJ: Maternal-fetal transfer of thyroxine in congenital hypothyroidism due to a total organification defect or thyroid agenesis. N Engl J Med 1989, 321:13-16.

11. Fisher DA: Fetal thyroid function: diagnosis and management of fetal thyroid disorders. Clin Obstet Gynecol 1997, 40:16-31.

12. Idris I, Srinivasan R, Simm A, Page RC: Maternal hypothyroidism in early and late gestation: effects on neonatal and obstetric outcome. Clin Endocrinol (Oxf) 2005, 63:560-565.

13. Sahu MT, Das V, Mittal S, Agarwal A, Sahu M: Overt and subclinical thyroid dysfunction among Indian pregnant women and its effect on maternal and fetal outcome. Arch Gynecol Obstet 2010, 281:215-220.
14. Chang SC, Thibodeaux JR, Eastvold ML, Ehresman DJ, Bjork JA, Froehlich JW, Lau C, Singh RJ, Wallace KB, Butenhoff JL: Thyroid hormone status and pituitary function in adult rats given oral doses of perfluorooctanesulfonate (PFOS). Toxicology 2008, 243:330-339.

15. Butenhoff JL, Stump DG, Parker GA, Wallace KB, Bjork JA, Ehresman DJ, Chang SC: Gestational and lactational exposure to potassium perfluorooctanesulfonate ( $\mathrm{K}+\mathrm{PFOS})$ in rats: toxicokinetics, thyroid hormone status, and related gene expression. Reproductive toxicology (Elmsford, N.Y) 2009, 27:387-399.

16. Lau C, Thibodeaux JR, Hanson RG, Rogers JM, Grey BE, Stanton ME, Butenhoff $J$, Stevenson LA: Exposure to perfluorooctane sulfonate during pregnancy in rat and mouse. II: postnatal evaluation. Toxicol Sci 2003, 74:382-392.

17. Thibodeaux JR, Hanson RG, Rogers JM, Grey BE, Barbee BD, Richards JH, Butenhoff $\lrcorner$, Stevenson LA, Lau C: Exposure to perfluorooctane sulfonate during pregnancy in rat and mouse. I: maternal and prenatal evaluations. Toxico/ Sci 2003, 74:369-381.

18. Knox SS, Jackson T, Frisbee SJ, Javins B, Ducatman AM: Perfluorocarbon exposure, gender and thyroid function in the C8 Health Project. J Toxicol Sci 2011, 36:403-410.

19. Dallaire $R$, Dewailly $E$, Pereg D, Dery S, Ayotte P: Thyroid function and plasma concentrations of polyhalogenated compounds in Inuit adults. Environ Health Perspect 2009, 117:1380-1386

20. Lin CY, Wen LL, Lin LY, Wen TW, Lien GW, Hsu SH, Chien KL, Liao CC, Sung FC, Chen PC, Su TC: The associations between serum perfluorinated chemicals and thyroid function in adolescents and young adults. J Hazard Mater 2013, 244-245:637-644.

21. Melzer D, Rice N, Depledge MH, Henley WE, Galloway TS: Association between serum perfluorooctanoic acid (PFOA) and thyroid disease in the U.S. National Health and Nutrition Examination Survey. Environ Health Perspect 2010, 118:686-692

22. Bloom MS, Kannan K, Spliethoff HM, Tao L, Aldous KM, Vena JE: Exploratory assessment of perfluorinated compounds and human thyroid function. Physiol Behav 2010, 99:240-245.

23. Chan E, Burstyn I, Cherry N, Bamforth F, Martin JW: Perfluorinated acids and hypothyroxinemia in pregnant women. Environ Res 2011, 111:559-564.

24. Emmett EA, Zhang H, Shofer FS, Freeman D, Rodway NV, Desai C, Shaw LM: Community exposure to perfluorooctanoate: relationships between serum levels and certain health parameters. J Occup Environ Med 2006, 48:771-779.

25. Kim S, Choi K, Ji K, Seo J, Kho Y, Park J, Kim S, Park S, Hwang I, Jeon J, Yang $\mathrm{H}$, Giesy JP: Trans-placental transfer of thirteen perfluorinated compounds and relations with fetal thyroid hormones. Environ Sci Technol 2011, 45:7465-7472.

26. Magnus P, Irgens LM, Haug K, Nystad W, Skjaerven R, Stoltenberg C: Cohort profile: the Norwegian Mother and Child Cohort Study (MoBa). Int J Epidemiol 2006, 35:1146-1150.

27. Whitworth KW, Haug LS, Baird DD, Becher G, Hoppin JA, Skjaerven R, Thomsen C, Eggesbo M, Travlos G, Wilson R, Longnecker MP: Perfluorinated compounds and subfecundity in pregnant women. Epidemiology (Cambridge, Mass) 2012, 23:257-263.

28. Ronningen KS, Paltiel L, Meltzer HM, Nordhagen R, Lie KK, Hovengen R, Haugen M, Nystad W, Magnus P, Hoppin JA: The biobank of the Norwegian Mother and Child Cohort Study: a resource for the next 100 years. Eur J Epidemiol 2006, 21:619-625.

29. Haug LS, Thomsen C, Becher G: A sensitive method for determination of a broad range of perfluorinated compounds in serum suitable for largescale human biomonitoring. J Chromatogr A 2009, 1216:385-393.

30. Weiland SK, Klenk J, Rzehak P, Richardson DB: Analyses of case-control data for additional outcomes. Epidemiology (Cambridge, Mass) 2007, 18:441-445.

31. Pearce EN, Oken E, Gillman MW, Lee SL, Magnani B, Platek D, Braverman LE: Association of first-trimester thyroid function test values with thyroperoxidase antibody status, smoking, and multivitamin use. Endocr Pract 2008, 14:33-39

32. Persky V, Turyk M, Anderson HA, Hanrahan LP, Falk C, Steenport DN, Chatterton R Jr, Freels S: The effects of PCB exposure and fish consumption on endogenous hormones. Environ Health Perspect 2001, 109:1275-1283.

33. del Escobar Rey F, Obregon MJ, de Morreale Escobar G: Is neuropsychological development related to maternal hypothyroidism or to maternal hypothyroxinemia? J Clin Endocrinol Metab 2000, 85:3975-3987. 
34. Yu WG, Liu W, Jin YH: Effects of perfluorooctane sulfonate on rat thyroid hormone biosynthesis and metabolism. Environ Toxicol Chem 2009, 28:990-996.

35. Weiss JM, Andersson PL, Lamoree MH, Leonards PE, van Leeuwen SP, Hamers T: Competitive binding of poly- and perfluorinated compounds to the thyroid hormone transport protein transthyretin. Toxicol Sci 2009, 109:206-216.

36. Mandel SJ, Spencer CA, Hollowell JG: Are detection and treatment of thyroid insufficiency in pregnancy feasible? Thyroid 2005, 15:44-53.

37. Fei C, Olsen J: Prenatal exposure to perfluorinated chemicals and behavioral or coordination problems at age 7 years. Environ Health Perspect 2011, 119:573-578.

38. Maisonet M, Terrell ML, McGeehin MA, Christensen KY, Holmes A, Calafat AM, Marcus M: Maternal concentrations of polyfluoroalkyl compounds during pregnancy and fetal and postnatal growth in British girls. Environ Health Perspect 2012, 120:1432-1437.

39. Soldin OP, Tractenberg RE, Hollowell JG, Jonklaas J, Janicic N, Soldin SJ: Trimester-specific changes in maternal thyroid hormone, thyrotropin, and thyroglobulin concentrations during gestation: trends and associations across trimesters in iodine sufficiency. Thyroid 2004 14:1084-1090

40. Soldin OP, Soldin D, Sastoque M: Gestation-specific thyroxine and thyroid stimulating hormone levels in the United States and worldwide. Ther Drug Monit 2007, 29:553-559.

41. Silvio R, Swapp KJ, La'ulu SL, Hansen-Suchy K, Roberts WL: Method specific second-trimester reference intervals for thyroid-stimulating hormone and free thyroxine. Clin Biochem 2009, 42:750-753.

42. Delange F: The disorders induced by iodine deficiency. Thyroid 1994 4:107-128.

43. Helgadottir LB, Skjeldestad FE, Jacobsen AF, Sandset PM, Jacobsen EM: Incidence and risk factors of fetal death in Norway: a case-control study. Acta obstetricia et gynecologica Scandinavica 2011, 90:390-397.

44. Krassas GE, Poppe K, Glinoer D: Thyroid function and human reproductive health. Endocr Rev 2010, 31:702-755.

45. Utiger RD: The thyroid: a fundamental and clinical text. Philadelphia: JB Lippincott; 1986.

46. Asvold BO, Bjoro T, Vatten $\mathrm{LJ}$ : Association of thyroid function with estimated glomerular filtration rate in a population-based study: the HUNT study. Eur J Endocrinol 2011, 164:101-105.

47. Loccisano AE, Longnecker MP, Campbell JL Jr, Andersen ME, 3rd Clewell HJ: Development of PBPK models for PFOA and PFOS for human pregnancy and lactation life stages. J Environ Sci Health [A]: Current, Issues 2013, 76:25-57.

48. Nilsen RM, Vollset SE, Gjessing HK, Skjaerven R, Melve KK, Schreuder P, Alsaker ER, Haug K, Daltveit AK, Magnus P: Self-selection and bias in a large prospective pregnancy cohort in Norway. Paediatr Perinat Epidemiol 2009, 23:597-608.

\section{Submit your next manuscript to BioMed Central and take full advantage of:}

- Convenient online submission

- Thorough peer review

- No space constraints or color figure charges

- Immediate publication on acceptance

- Inclusion in PubMed, CAS, Scopus and Google Scholar

- Research which is freely available for redistribution 